\title{
Prognostic value of plasma fibrinogen in hepatocellular carcinoma: a meta-analysis
}

This article was published in the following Dove Press journal:

Cancer Management and Research

\author{
Guanqun Huang ${ }^{1, *}$ \\ Hui Jiang $2, *$ \\ Ye $\operatorname{Lin}^{3}$ \\ Yanpeng $\mathrm{Wu}^{1}$ \\ Weilong Cai' \\ Boyun Shi ${ }^{2}$ \\ Yuanwei Luo' \\ Zhixiang Jian ${ }^{3}$ \\ Xinke Zhou ${ }^{2}$
}

'Department of General Surgery, The Fifth Affiliated Hospital of Guangzhou Medical University, Guangzhou, Guangdong 510700, People's Republic of China; ${ }^{2}$ Department of Abdominal Oncology, The Fifth Affiliated Hospital of Guangzhou Medical University, Guangzhou, Guangdong 5 I 0700, People's Republic Surgery, Guangdong General Hospital, Guangdong Academy of Medical Sciences, Guangzhou, Guangdong 510080, People's Republic of China

*These authors contributed equally to this work

Correspondence: Zhixiang Jian

Department of General Surgery,

Guangdong General Hospital, Guangdong

Academy of Medical Sciences, 106

Zhongshan Er Road, Guangzhou 510080,

People's Republic of China

Tel +86 I 3802902850

Email jhgywy_1314@163.com

Xinke Zhou

Department of Abdominal Oncology, The Fifth Affiliated Hospital of Guangzhou

Medical University, 62I Gangwan Road,

Guangzhou 510700, People's Republic

of China

$\mathrm{Tel}+86$ I 37। 9199820

Fax +86 2082297393

Email gywyzxk_119@163.com of China; ${ }^{3}$ Department of General

Background: Elevated plasma fibrinogen levels have been associated with tumor progression in several malignancies. Our study aims to characterize the clinical significance of elevated plasma fibrinogen levels in patients with hepatocellular carcinoma (HCC).

Materials and methods: Relevant published articles were systematically searched in electronic databases including PubMed, Embase, and Web of Science. The pooled differences in plasma fibrinogen levels among $\mathrm{HCC}$, cirrhotic, and control groups were expressed as weighted mean differences (WMDs) and their corresponding 95\% CIs. The associations between elevated fibrinogen and overall survival (OS) and disease-free survival (DFS)/recurrence-free survival (RFS) were expressed as HRs and their 95\% CIs, whereas the associations between elevated fibrinogen and various types of clinical characteristic of patients with HCC were expressed as ORs and their corresponding 95\% CIs.

Results: Results showed that the plasma fibrinogen levels in patients with HCC were not significantly different than that in healthy controls (WMD $=0.50,95 \% \mathrm{CI}=[-0.82,1.82]$, $P=0.457)$ or patients with cirrhosis $(\mathrm{WMD}=-0.62,95 \% \mathrm{CI}=[-1.56,0.33], P=0.200)$. However, our results showed that compared to those with normal levels, patients with HCC and elevated plasma fibrinogen levels showed poorer OS $(\mathrm{HR}=2.08,95 \% \mathrm{CI}=[1.67,2.59]$, $P<0.0001)$ and DFS/RFS $(\mathrm{HR}=1.90,95 \% \mathrm{CI}=[1.52,2.37], P<0.0001)$. Results of trial sequential analysis of the OS indicated that currently available studies were sufficient to validate the negative prognostic value of elevated plasma fibrinogen in patients with HCC. Clinicopathological analyses showed that high plasma fibrinogen levels were associated with tumor progression as indicated by advanced tumor stage, larger tumor size, increased tumor number, and the presence of vascular invasion.

Conclusion: Elevated plasma fibrinogen levels are associated with poor prognosis and advanced tumor progression. Plasma fibrinogen may serve as a negative prognostic biomarker in patients with HCC.

Keywords: hepatocellular carcinoma, fibrinogen, prognosis, tumor progression, meta-analysis

\section{Introduction}

Hepatocellular carcinoma (HCC) is a primary malignancy of the liver and is now the second leading cause of cancer deaths worldwide, following lung cancer. ${ }^{1}$ The global incidence of $\mathrm{HCC}$ is still on the rise with the highest prevalence in East Asia, ${ }^{1}$ where the mortality rate is 33.5 per 100,000 people. ${ }^{2}$ The majority of $\mathrm{HCC}$ cases occur with underlying chronic hepatitis $\mathrm{B}$ or $\mathrm{C}$ viral infection and cirrhosis. ${ }^{3,4}$ The other main risk factors for HCC are alcohol intoxication and aflatoxin. ${ }^{3,5}$ Current treatment modalities include radical therapies (eg, surgical resection, liver transplantation, radiofrequency ablation) and adjuvant therapies (eg, transarterial chemoembolization [TACE], systemic 
chemotherapy, sorafenib). Although these methods have significantly improved the long-term survival of patients with HCC, the overall prognosis is largely limited by the extent of liver dysfunction. ${ }^{6}$ Although various tumor staging systems are available to predict prognosis, they too have major limitations. For instance, the Milan criteria are solely based on the preoperative imaging without consideration of molecular signatures ${ }^{7}$ and biopsy has the risk of introducing tumor cell seeding. ${ }^{8}$ Therefore, it would be useful to identify effective preoperative prognostic biomarkers that may facilitate outcome prediction and indicate the requirement for aggressive therapy in high-risk patients with HCC.

To date, the most common serum biomarker used by clinicians to diagnose HCC is alpha-fetoprotein (AFP). However, the reliability of AFP is questionable due to its lack of sensitivity and specificity. ${ }^{9}$ In addition, AFP marker is less valuable in the early stages of $\mathrm{HCC}$ when the tumor size is less than $3 \mathrm{~cm} .{ }^{10}$ Therefore, new and more effective prognostic serum biomarkers are needed to improve clinical outcomes.

Fibrinogen is a $340-\mathrm{kDa}$ soluble plasma glycoprotein synthesized in the liver by hepatocytes. It is an important coagulation factor. In the coagulation cascade, fibrinogen is enzymatically converted to fibrin by activated thrombin and subsequently forms a clot. In situations such as tissue injury, infection, and inflammation, fibrinogen concentrations are rapidly elevated. ${ }^{11,12}$ Recently, emerging evidence has associated the elevation of fibrinogen level with tumor progression in several malignancies, such as breast cancer, ${ }^{13}$ gallbladder cancer, ${ }^{14}$ gastric cancer, ${ }^{15}$ colorectal cancer, ${ }^{16}$ and lung cancer. ${ }^{17}$ Few studies have also reported that patients with HCC and elevated pretreatment plasma fibrinogen levels have poor outcomes. ${ }^{18,19}$ However, these studies were limited in size, varied in cutoff concentrations, or administered heterogenous treatments, and the correlations between elevated fibrinogen and clinicopathological parameters of HCC were unclear. In addition, there are conflicting findings on the levels of plasma fibrinogen in HCC patients compared to that in control or cirrhotic groups. Several studies reported that HCC patients showed higher levels of pretreatment plasma fibrinogen compared to healthy controls or cirrhotic patients. ${ }^{20-22}$ However, Basili et $\mathrm{al}^{23}$ and Liu et $\mathrm{al}^{24}$ found no significant difference in the plasma fibrinogen levels between HCC and control or cirrhotic groups, and Hessien et $\mathrm{al}^{25}$ indicated lower levels of fibrinogen in HCC patients compared to that in control and cirrhotic patients. Therefore, our present study aims to summarize the currently available studies to examine 1) whether the pretreatment plasma fibrinogen levels were significantly different among HCC, cirrhotic, and healthy subjects; 2) the association between elevated plasma fibrinogen and prognosis in patients with HCC; and 3 ) the association between elevated fibrinogen levels and tumor progression in patients with HCC. To the best of our knowledge, no meta-analysis has been performed to assess elevated plasma fibrinogen risk in patients with HCC.

\section{Materials and methods}

This meta-analysis was conducted in accordance with the PRISMA guidelines. The studies included in this metaanalysis are previously published, and therefore, ethical approval and informed consent are not required.

\section{Literature search strategy}

Relevant published literatures were systematically searched in electronic databases including PubMed, Embase, and Web of Science. The last update was September 2017. The keywords used included "Fibrinogen", "liver neoplasm", and "hepatocellular carcinoma". Specifically, the keywords used in PubMed were (Fibrinogen) AND (neoplasm, liver [MeSH Terms] OR hepatocellular carcinoma). In Embase, the keywords used were Quick search: Fibrinogen AND (liver neoplasm OR hepatocellular carcinoma). In Web of Science, the keywords used were Fibrinogen AND (liver neoplasm OR hepatocellular carcinoma) [Topic]. There were no restrictions to the search process in terms of language, date, research design, or publication status.

\section{Study selection criteria}

Studies were included when they met the following inclusion criteria:

1. diagnosis of HCC was proven by pathological methods;

2. pretreatment plasma fibrinogen was measured in all participants;

3. mean and SD of pretreatment plasma fibrinogen levels were reported in patients with $\mathrm{HCC}$, cirrhosis, and/or healthy controls;

4. complete clinicopathological characteristics were evaluated prior to treatment; and

5. outcomes included overall survival (OS), disease-free survival (DFS), and/or recurrence-free survival (RFS).

Studies were excluded when they met the following exclusion criteria:

1. experiments were performed in vitro or in vivo;

2. study designs were case reports and reviews;

3. fibrinogen peptide chains, mRNA or protein expression, or fibrinogen degradation products were measured; 
4. conference proceedings that contained only summaries;

5. articles were not in English or Chinese; and

6. literature with data that could not be extracted.

\section{Outcomes of interest}

The primary outcomes of interest were OS, DFS, and RFS. Another outcome of interest was the association between fibrinogen levels and clinicopathological parameters. Other outcomes of interest were pretreatment plasma fibrinogen levels of HCC, cirrhotic, and/or healthy subjects.

\section{Data extraction and quality assessment}

Two independent researchers performed literature selection, evaluation, and extraction. Data on the following characteristics were retrieved: first author; year of publication; country in which the study was conducted; number of HCC, cirrhotic, and control subjects enrolled; age; sex; pretreatment plasma fibrinogen level measurements and methods; plasma fibrinogen cutoff values; methods used to determine cutoff values; treatments received; and follow-up period. Discrepancies were resolved by discussion to consensus. If both univariate and multivariate outcomes were provided in an article, we chose the latter. Efforts were made to contact the corresponding authors for any information not reported in the article.

The Newcastle-Ottawa Scale (NOS) was used to assess the quality of the studies included. NOS employs a "star" rating system that evaluates eight items, which are categorized into three domains: selection (four items: maximum one star each), comparability (one item: maximum two stars), and outcome (three items: maximum one star each). The NOS assessment, which ranges from 0 (worst) to 9 (best) stars, provides a semiquantitative appraisal for the overall quality of each study. Studies with scores of 6 or higher were regarded as high quality.

\section{Statistical analyses}

All statistical analyses were performed using Stata 13.1 (StataCorp LP, College Station, TX, USA). The pooled differences in plasma fibrinogen levels among HCC, cirrhotic, and control groups were expressed as weighted mean differences (WMDs) and their corresponding 95\% CIs. The associations between elevated fibrinogen and OS or DFS/ RFS were expressed as the HRs and their corresponding 95\% CIs, and the associations between elevated fibrinogen and various types of clinical characteristics of patients with HCC were expressed as ORs and their corresponding 95\% CIs. Heterogeneity among studies was assessed using Cochran's
Q test and Higgins $I^{2}$ statistic. ${ }^{26}$ If no significant heterogeneity was indicated among studies $\left(P>0.1, I^{2}<50 \%\right)$, a fixed effect model was applied for analysis. ${ }^{27}$ Otherwise, the random effect model was adopted. ${ }^{28}$ Sensitivity analysis was performed using sequential omission of individual studies to evaluate the influence of an individual study on the overall pooled estimates. If the pooled estimates were affected after removal, it indicated that the results were inconsistent. Egger's linear regression tests were used to assess for publication bias, and any indication of publication bias was further tested with trim and fill analysis. $P<0.05$ indicated significant bias.

Trial sequential analysis (TSA) was conducted to minimize type I error, evaluate required information size, and assess whether current available studies were sufficient for a firm conclusion. The required information size refers to the required number of participants to produce statistically significant result in meta-analysis. The diversity-adjusted required information size of 784 patients was calculated on the basis of type I error of 5\%, type II error of $20 \%$, the control event proportion of $40 \%$, and a relative risk reduction of $20 \%$ in outcomes. When the cumulative Z-curve crossed the trial sequential monitoring boundary for benefit, harm or futility, or the required information size, this result suggests conclusive evidence within this meta-analysis. Otherwise, more clinical studies are warranted to reach a consistent conclusion.

\section{Results \\ Study selection process}

Figure 1 depicts the fully detailed process of article search and selection. The electronic database searches yielded a total of 1,128 potential articles (PubMed $=410$, Embase $=565$, and Web of Science $=153)$. The titles and abstracts were screened, and 1,094 articles were excluded because they covered irrelevant research topics $(n=1,019)$, lacked full text $(n=20)$, or were duplicates $(n=55)$. Thirty-four fulltext articles were retrieved for detailed evaluation. Eighteen articles were eliminated due to the following reasons: in vivo/ in vitro study $(n=2)$; case report $(n=2)$; study that measured fibrinogen peptide chains, mRNA or protein expression, or fibrinogen degradation product $(n=12)$; cases that included other primary liver tumor besides HCC $(\mathrm{n}=1)$; and data not presented as mean $\pm \operatorname{SD}(n=1)$. A total of 16 articles were included in this study: eight articles measured and compared the plasma fibrinogen levels among HCC, cirrhotic, and healthy subjects, and eight articles studied the clinicopathological and prognostic value of plasma fibrinogen in HCC. 


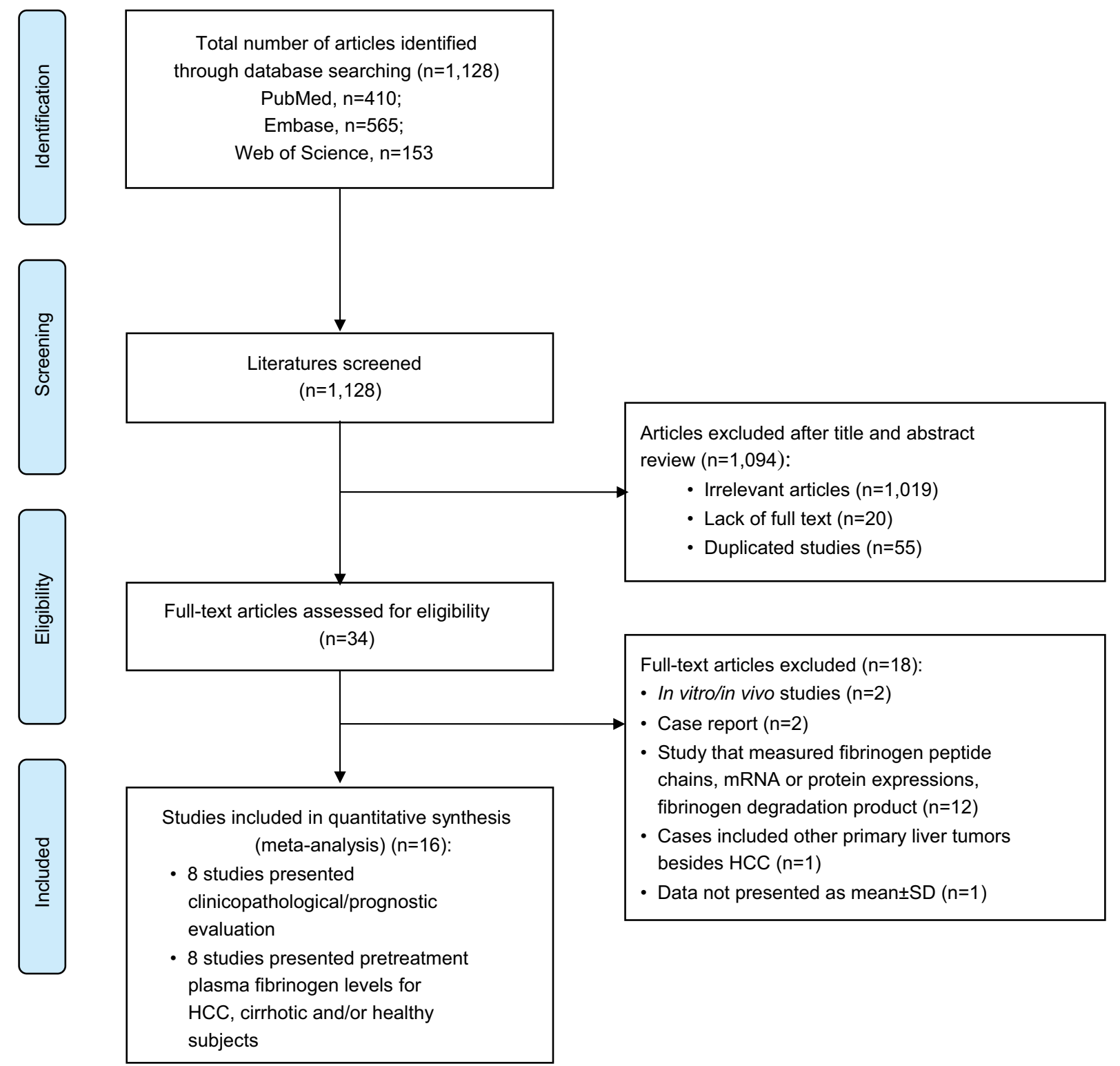

Figure I Flow chart of article search and selection.

Note: PLoS Medicine (OPEN ACCESS) Moher D, Liberati A, Tetzlaff J, Altman DG, The PRISMA Group (2009). Preferred Reporting Items for Systematic Reviews and MetaAnalyses: The PRISMA Statement. PLoS Med 6(7): el 000097 . doi: 10.137I/journal.pmed I000097.55

Abbreviation: HCC, hepatocellular carcinoma.

\section{Study characteristics and quality assessment}

Eight studies that reported OS, DFS, or RFS were included in the meta-analysis to assess the prognostic value of plasma fibrinogen in patients with HCC. The main characteristics of the included studies are presented in Table 1. All studies were conducted in Asia, involving a total of 1,961 patients with HCC. Pretreatment plasma fibrinogen levels were measured using Clauss assay or serum biochemistry method. The cutoff values for plasma fibrinogen level were determined by different methods, including receiver operating characteristics (ROC), clinical reference range, or median. The cutoff values divided patients with HCC into an elevated plasma fibrinogen group and a normal plasma fibrinogen group. All patients with $\mathrm{HCC}$ also received either curative treatment or palliative treatment. In addition, eight studies were included to examine the pretreatment plasma fibrinogen levels in HCC, cirrhotic, and/ or healthy subjects. Six studies presented plasma fibrinogen levels in patients with $\mathrm{HCC}$ and healthy controls, three studies presented data for patients with cirrhosis and controls, and six studies presented data for patients with $\mathrm{HCC}$ and patients with cirrhosis. The details of the studies are provided in Table 2.

We also used the NOS to evaluate the quality of the eight studies that reported OS, DFS, and RFS. All of the studies 


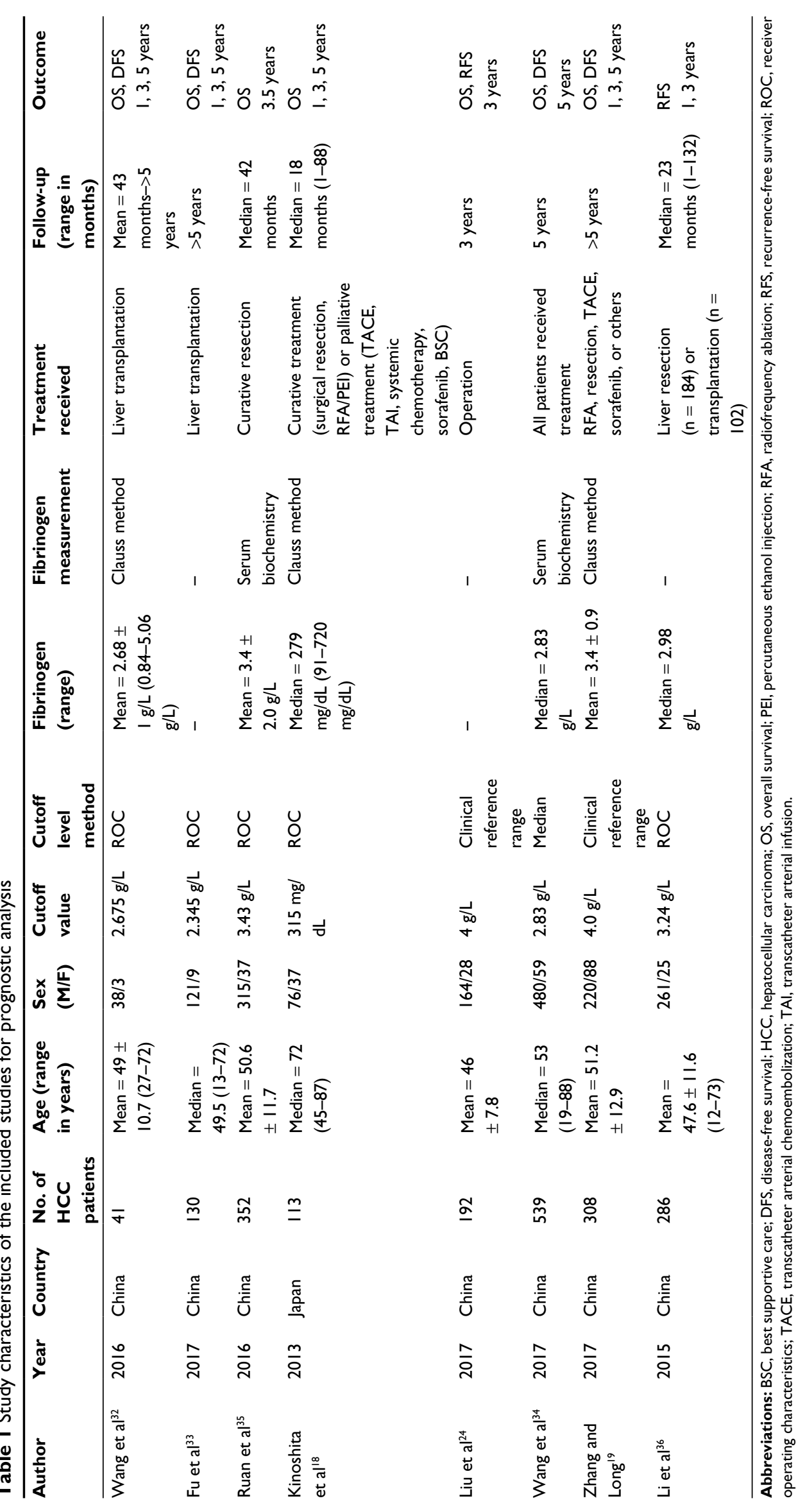


Table 2 Baseline plasma fibrinogen levels of HCC, cirrhotic, and healthy subjects

\begin{tabular}{|c|c|c|c|c|c|c|c|c|c|}
\hline \multirow[t]{2}{*}{ Study } & \multirow[t]{2}{*}{ Year } & \multirow[t]{2}{*}{ Country } & \multirow{2}{*}{$\begin{array}{l}\text { No. of } \\
\text { controls }\end{array}$} & \multirow{2}{*}{$\begin{array}{l}\text { No. of } \\
\text { HCC } \\
\text { subjects }\end{array}$} & \multirow{2}{*}{$\begin{array}{l}\text { No. of } \\
\text { cirrhotic } \\
\text { subjects }\end{array}$} & \multicolumn{3}{|c|}{ Plasma fibrinogen level (g/L) } & \multirow[t]{2}{*}{ Method } \\
\hline & & & & & & Controls & $\begin{array}{l}\text { HCC } \\
\text { subjects }\end{array}$ & $\begin{array}{l}\text { Cirrhotic } \\
\text { subjects }\end{array}$ & \\
\hline Zhu et $\mathrm{al}^{22}$ & 2009 & China & 30 & 114 & - & $2.90 \pm 0.46$ & $3.75 \pm 1.41$ & - & $\begin{array}{l}\text { COULTER ACL-200 } \\
\text { automated coagulant analyzer }\end{array}$ \\
\hline Alkim et $\mathrm{a}^{29}$ & 2012 & Turkey & 10 & 11 & 13 & $2.6 \pm 0.4$ & $2.3 \pm 0.5$ & $2.4 \pm 0.6$ & Conventional method \\
\hline $\begin{array}{l}\text { Hessien } \\
\text { et } \mathrm{a}^{25}\end{array}$ & 2015 & Egypt & 10 & 10 & 10 & $2.74 \pm 0.44$ & $0.99 \pm 0.28$ & $1.91 \pm 0.43$ & $\begin{array}{l}\text { Clauss method using } \\
\text { commercially available kit }\end{array}$ \\
\hline Doğan et al ${ }^{21}$ & 1997 & Turkey & - & 20 & 44 & - & $3.51 \pm 1.30$ & $2.36 \pm 1.08$ & Standard method \\
\hline Basili et $\mathrm{a}^{23}$ & 1997 & Italy & - & 18 & 18 & - & $2.30 \pm 1.45$ & $\mathrm{I} .8 \mathrm{I} \pm 0.72$ & $\begin{array}{l}\text { Clauss method employing the } \\
\text { ortho quantitative fibrinogen } \\
\text { assay }\end{array}$ \\
\hline Casaril et al ${ }^{20}$ & 1989 & Italy & - & 49 & 110 & - & $4.25 \pm 1.80$ & $2.64 \pm 1.05$ & Routine laboratory screening \\
\hline $\begin{array}{l}\text { Al Ghumlas } \\
\text { et a }\left.\right|^{30}\end{array}$ & 2005 & Saudi Arabia & 40 & 13 & 67 & $\begin{array}{l}3.07 \pm 1.17 \\
g / L\end{array}$ & $4.58 \pm 2.06$ & $2.83 \pm 1.44$ & $\begin{array}{l}\text { Turbidimetric method of Ellis } \\
\text { and Stransky }\end{array}$ \\
\hline $\begin{array}{l}\text { van der Walt } \\
\text { et a }\left.\right|^{31}\end{array}$ & 1977 & South Africa & 19 & 19 & - & $3.15 \pm 0.76$ & $5.83 \pm 2.78$ & - & Ellis and Stransky \\
\hline
\end{tabular}

Note: Data are presented as mean \pm SD.

Abbreviation: HCC, hepatocellular carcinoma.

scored between 8 and 9 , indicating that the quality of the included studies was high (Table 3).

\section{Plasma fibrinogen levels among HCC, cirrhotic, and healthy subjects}

We examined the plasma fibrinogen levels in HCC, cirrhotic, and healthy subjects. A random effect model showed no significant difference in the plasma fibrinogen levels among patients with HCC and healthy controls (WMD $=0.50,95 \% \mathrm{CI}=[-0.82,1.82], P=0.457$; Figure $2 \mathrm{~A})$. However, compared to healthy individuals, patients with cirrhosis had significantly lower plasma fibrinogen levels (WMD $=-0.44,95 \% \mathrm{CI}=[-0.86,-0.01], P=0.043$; Figure 2B). The plasma fibrinogen levels were not significantly different between patients with HCC and patients with cirrhosis $(\mathrm{WMD}=-0.62,95 \% \mathrm{CI}$, $[-1.56,0.33], P=0.200$; Figure 2C).

\section{Association between plasma fibrinogen and HCC prognosis}

\section{Correlation between elevated plasma fibrinogen and OS}

Meta-analysis of the six OS-related studies showed little heterogeneity among studies $\left(I^{2}=25.7 \%, P=0.242\right)$. A fixed effect model showed that patients with $\mathrm{HCC}$ and high pretreatment plasma fibrinogen levels had significantly poorer OS compared to those with normal fibrinogen levels ( $\mathrm{HR}=$ $2.08,95 \% \mathrm{CI}=[1.67,2.59], P<0.0001$; Figure 3A). Sensitivity analysis showed that the pooled result was relatively stable
(Figure 3B). Egger's test $(P=0.008)$ showed the presence of publication bias. Further, trim and fill analysis showed that after adjusting for three studies, the recalculated pooled HR for OS was $1.85(95 \% \mathrm{CI}=[1.51,2.27]$; Figure 3C), which was not significantly changed from the initial estimate (HR $=2.08,95 \% \mathrm{CI}=[1.67,2.59])$. Our data suggest that the presence of publication bias has no significant effect on the overall finding.

We also performed TSA meta-analysis on the OS results. Although the number of participants had not reached the required information size, our TSA showed that the cumulative Z-curve crossed the trial sequential monitoring boundary for harm, confirming the poorer survival rate among patients with HCC and high levels of plasma fibrinogen. Our results suggest that currently available studies are sufficient to conclude the negative prognostic value of elevated plasma fibrinogen in patients with HCC (Figure 4).

\section{Correlation between elevated plasma fibrinogen and DFS/RFS}

Meta-analysis of the 5 DFS-/RFS-related studies showed low heterogeneity among studies $\left(I^{2}=36.3 \%, P=0.179\right)$. A fixed effect model showed that patients with HCC and high pretreatment plasma fibrinogen levels had significantly poorer outcomes compared to those with normal fibrinogen levels $(\mathrm{HR}=1.90,95 \% \mathrm{CI}=[1.52,2.37], P<0.0001$; Figure $5 \mathrm{~A})$. Sensitivity analysis showed that the pooled result was relatively stable (Figure 5B). Egger's test $(P=0.095)$ showed no publication bias. 


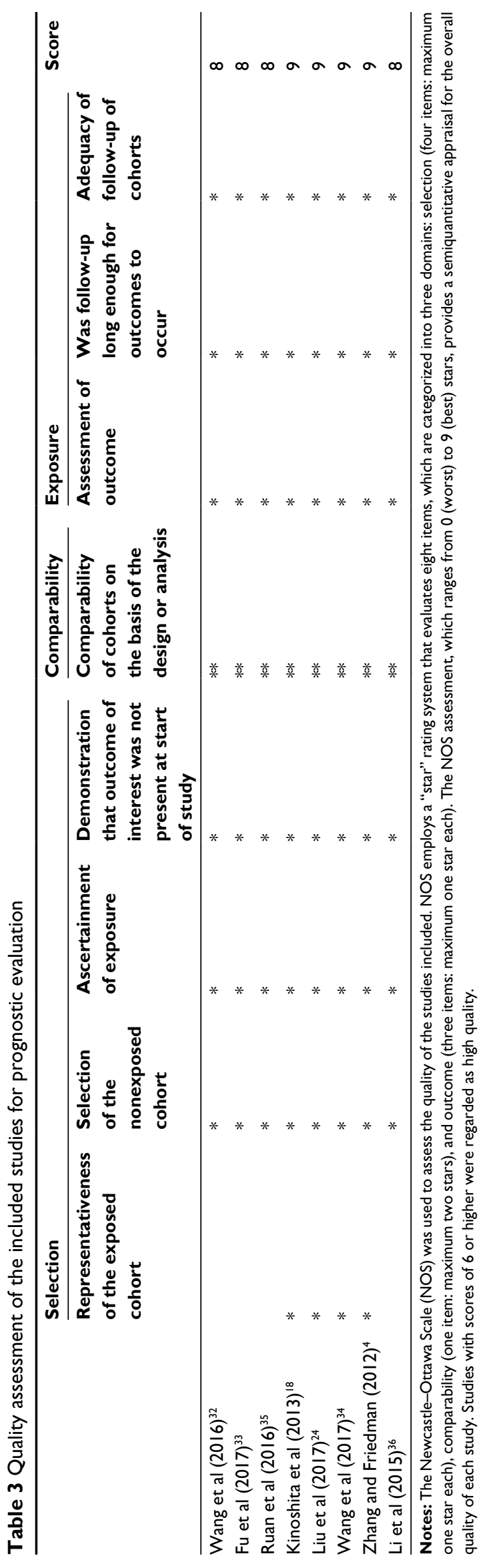

\section{Association between plasma fibrinogen and clinicopathological parameters of HCC}

Clinicopathological analyses showed that elevated plasma fibrinogen levels were significantly associated with advanced tumor stage (stage III-IV vs stage I-II, OR $=2.37,95 \%$ $\mathrm{CI}=[1.83,3.07], P<0.00001)$, larger tumor size $(\geq 5 \mathrm{vs}$ $<5 \mathrm{~cm}, \mathrm{OR}=3.04,95 \% \mathrm{CI}=[2.22,4.15], P<0.00001)$, increased tumor number (multiple vs single, $\mathrm{OR}=1.54$, $95 \% \mathrm{CI}=[1.17,2.05], P=0.002 ;>3$ vs $\leq 3$ tumor nodules, $\mathrm{OR}=1.99,95 \% \mathrm{CI}=[1.02,3.87], P=0.04)$, and vascular invasion $(\mathrm{OR}=3.81,95 \% \mathrm{CI}=[2.25,6.44], P<0.00001)$. Plasma fibrinogen levels were significantly higher in patients with HCC and mild cirrhosis (Child A) compared to those with moderate or advanced cirrhosis (Child B/C) (Child A vs Child $\mathrm{B} / \mathrm{C}, \mathrm{OR}=1.53,95 \% \mathrm{CI}=[1.01,2.33], P=0.04)$. Higher plasma fibrinogen was also marginally associated with increased serum AFP level $(\mathrm{OR}=1.37,95 \% \mathrm{CI}=[1.00$, $1.86], P=0.05)$. No significant association was found when evaluating age, sex, hepatitis B surface antigen (HBsAg), and alcohol use. The correlations between plasma fibrinogen and the clinical features of HCC are depicted in Figure 6 and summarized in Table 4.

\section{Discussion}

In this meta-analysis, we identified eight studies ${ }^{20-23,25,29-31}$ that examined the pretreatment plasma fibrinogen levels in HCC, cirrhotic, and/or healthy subjects. We observed that patients with cirrhosis had significantly lower plasma fibrinogen levels compared to healthy controls (WMD = $-0.44,95 \% \mathrm{CI}=[-0.86,-0.01], P=0.043)$. Fibrinogen is a product of hepatocytes. Therefore, patients with cirrhosis and compromised liver function may generally have less plasma fibrinogen levels than healthy counterparts. ${ }^{25,29}$ However, our WMD analyses showed that the pretreatment plasma fibrinogen levels in HCC cases were not significantly elevated when compared with that in healthy subjects (WMD $=0.50,95 \% \mathrm{CI}$ $=[-0.82,1.82], P=0.457$ ) or patients with cirrhosis (WMD $=-0.62,95 \% \mathrm{CI}=[-1.56,0.33], P=0.200)$. Therefore, plasma fibrinogen level is not usable as a diagnostic marker for HCC detection.

On the other hand, our prognostic value analyses, which included eight studies ${ }^{18,19,24,32-36}$ with a total number of 1,961 patients with HCC, demonstrated that those patients with HCC and elevated fibrinogen levels are more likely to experience poorer $\mathrm{OS}(\mathrm{HR}=2.08,95 \% \mathrm{CI}=[1.67,2.59]$, $P<0.0001)$ and recurrence $(\mathrm{HR}=1.90,95 \% \mathrm{CI}=[1.52$, 2.37], $P<0.0001)$. Furthermore, TSA indicated that currently available studies are sufficient to validate the negative 
A $\mathrm{HCC}$ versus control

Study

WMD $(95 \% \mathrm{Cl}) \quad$ Weight

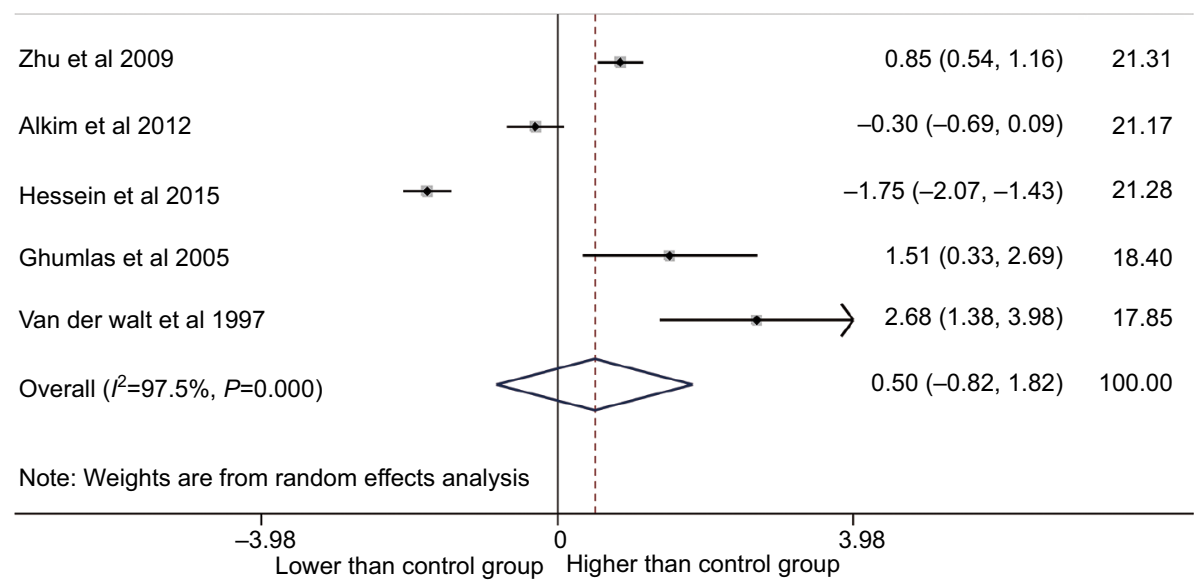

B Cirrhosis versus control

Study

WMD $(95 \% \mathrm{Cl}) \quad$ Weight

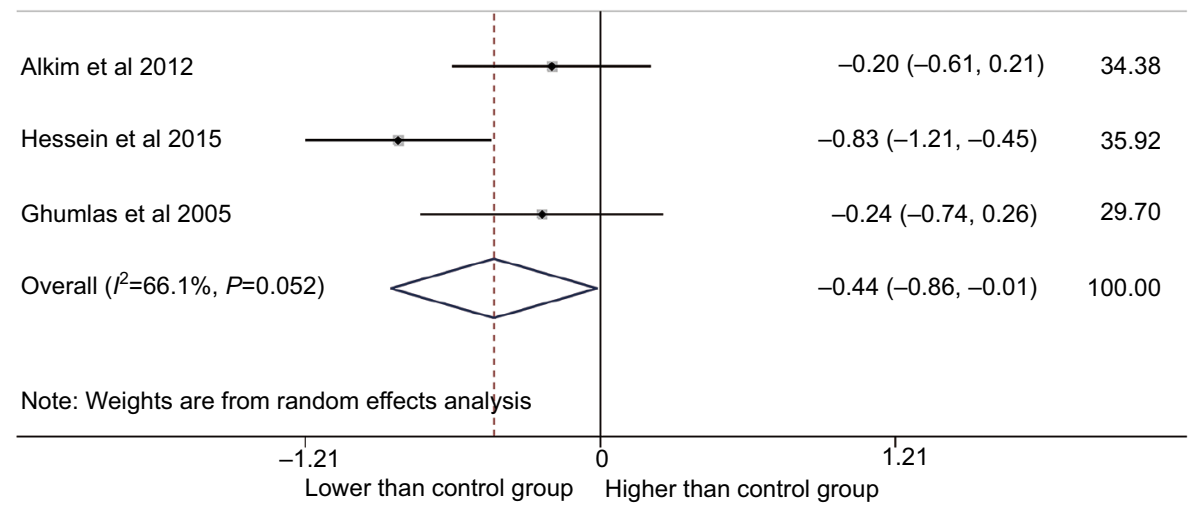

C HCC versus control

$\%$

Study

WMD $(95 \% \mathrm{Cl}) \quad$ Weight

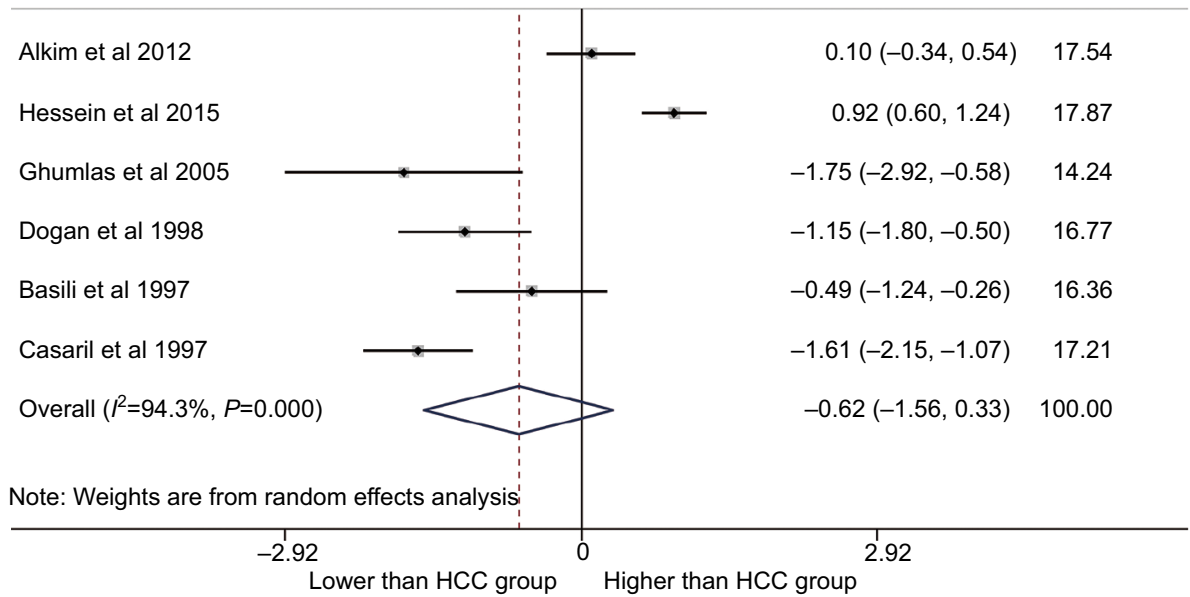

Figure 2 Forest plot for plasma fibrinogen levels in (A) patients with HCC compared to that in healthy controls, (B) cirrhotic patients compared to healthy controls, and (C) patients with $\mathrm{HCC}$ compared to patients with cirrhosis.

Abbreviations: HCC, hepatocellular carcinoma; WMD, weighted mean difference. 
A Overall survival

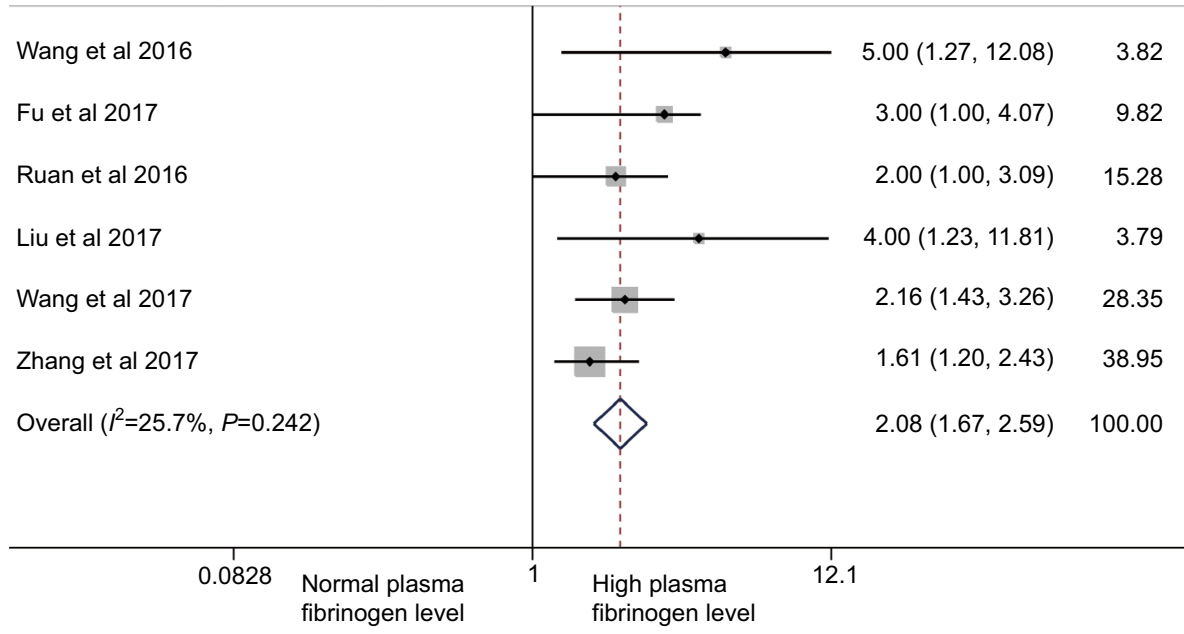

B Sensitivity analysis

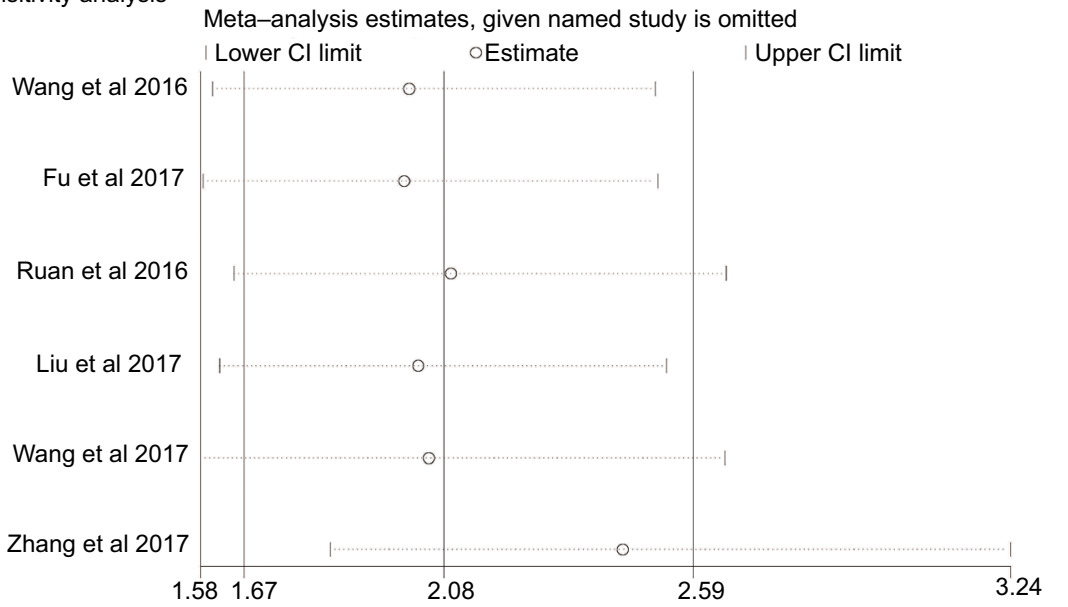

C Trim and fill analysis

Filled funnel plot pseudo $95 \%$ confience limits

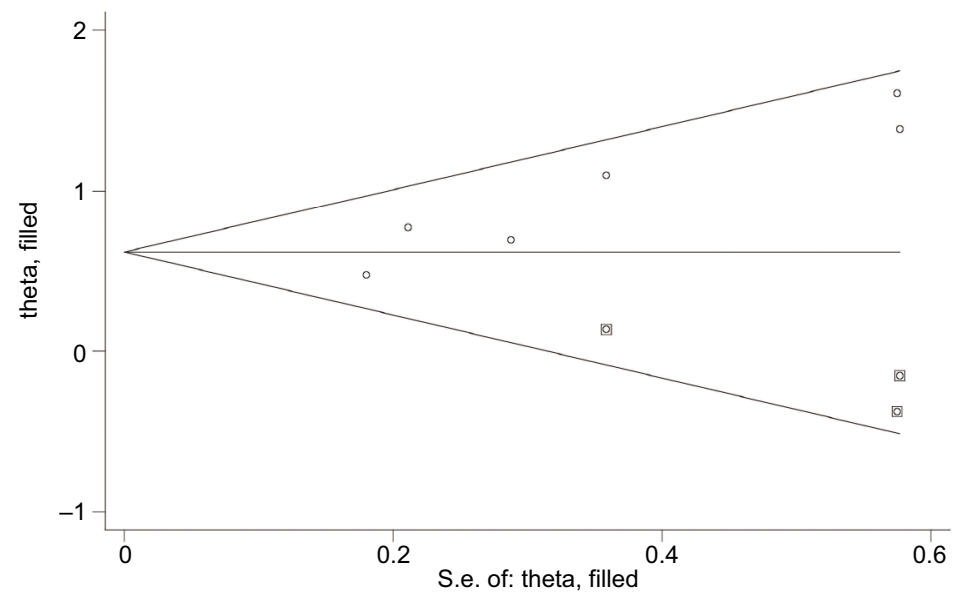

Figure 3 Association of elevated plasma fibrinogen level with overall survival in patients with hepatocellular carcinoma.

Notes: (A) Forest plot of HRs for overall survival. (B) Sensitivity analysis of overall survival. (C) Trim and fill analysis for the overall survival. The squares represent the adjusted studies. 


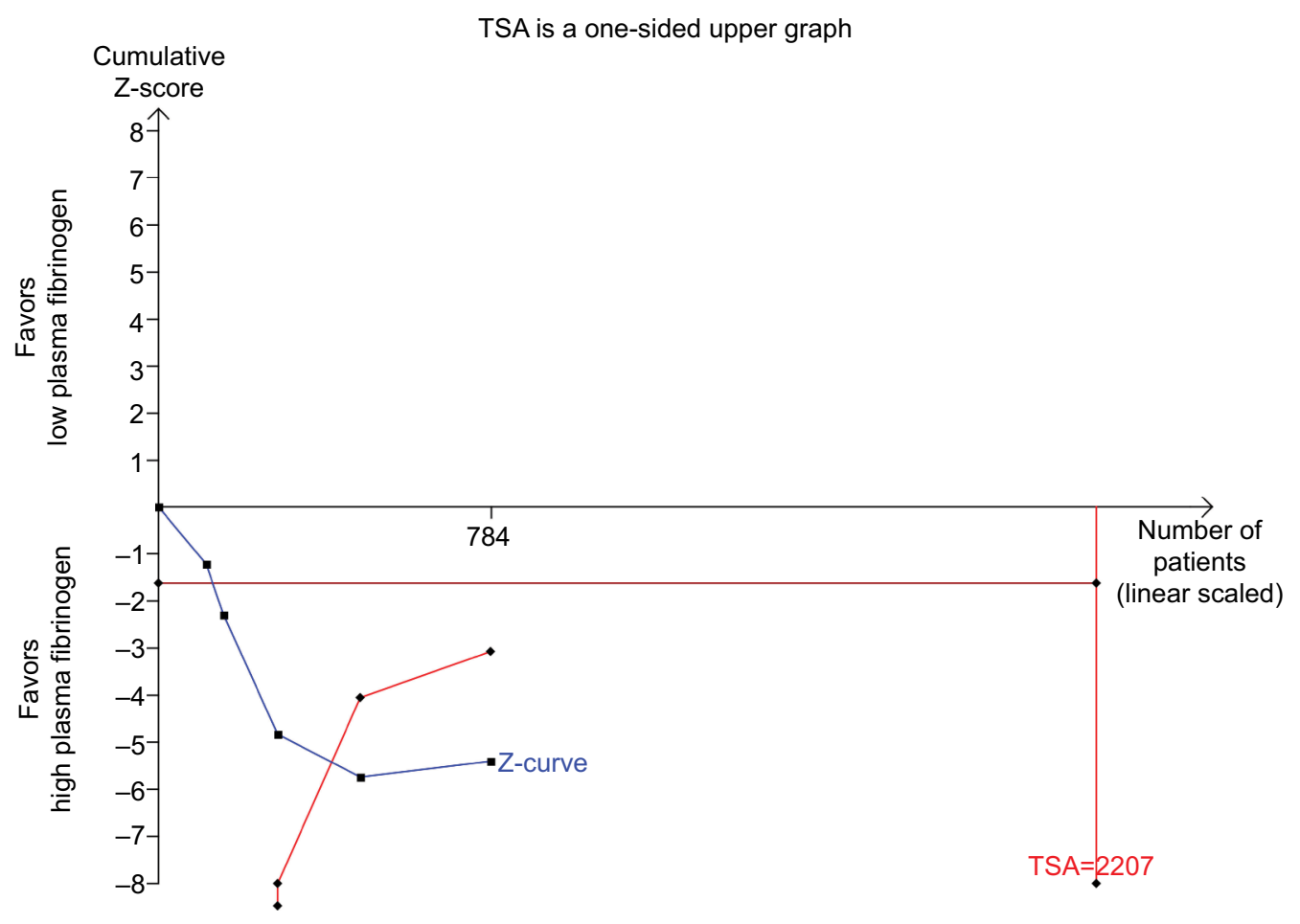

Figure 4 TSA of the association between elevated plasma fibrinogen levels and overall survival in patients with hepatocellular carcinoma.

Notes: The diversity-adjusted required information size of 784 patients was calculated on the basis of type I error of $5 \%$, type II error of $20 \%$, the control event proportion of $40 \%$, and a relative risk reduction of $20 \%$ in outcomes. The cumulative Z-curve crossed the trial sequential monitoring boundary for harm, confirming the poorer survival rate in patients with hepatocellular carcinoma and high plasma fibrinogen.

Abbreviation: TSA, trial sequential analysis.

prognostic value of elevated plasma fibrinogen levels in patients with HCC. Our clinicopathological analyses also indicated that patients with HCC and elevated plasma fibrinogen levels are likely to be associated with a higher pathological stage including advanced tumor stage, larger tumor size, increased tumor number, and the presence of vascular invasion, implicating that plasma fibrinogen level is closely related to tumor progression.

In addition to our analysis, recent studies also indicated a positive correlation between elevated plasma fibrinogen levels and poorer prognosis in other solid tumors including esophageal carcinoma, ${ }^{37-39}$ small cell lung carcinoma, ${ }^{40}$ nonsmall cell lung carcinoma, ${ }^{41}$ upper urinary tract urothelial carcinoma, ${ }^{42,43}$ epithelial ovarian cancer, ${ }^{44}$ colorectal cancer, ${ }^{45}$ renal cell carcinoma, ${ }^{46,47}$ and gastric cancer. ${ }^{48,49}$ There are several theories that may explain this association: 1) tumor progression is usually coupled with elevated inflammatory responses and leukocyte infiltration into tumor stroma. Infiltrated leukocytes may convert fibrinogen into fibrin matrix, which encapsulates the tumor cells and promotes tumor angiogenesis; ${ }^{50}$ 2) fibrinogen may serve as an extracellular matrix protein that regulates the growth of cancer cells by binding to growth factors (eg, vascular endothelial growth factor, fibroblast growth factor-2, platelet-derived growth factor) and promote cellular adhesion, proliferation, and metastasis; and ${ }^{51-53} 3$ ) fibrinogen promotes the adhesion of tumor cells to platelets, which leads to platelet aggregation and thrombin formation around tumor cells. The tumorassociated platelets and thrombin protect tumor cells against the attack of natural killer cells..$^{54}$ Moreover, in vitro studies also suggested that high levels of fibrinogen induced epithelial-mesenchymal transition, which is a hallmark for cancer invasion and metastasis. ${ }^{14}$

As a plasma biomarker, fibrinogen has its advantages: blood sampling is a minimally invasive method that patients tolerate well, and it allows replicated sampling for long-term surveillance. Plasma fibrinogen levels can be determined easily by using routine coagulation parameters tested in the laboratory, which is rapid and cost-effective compared to other measurements, such as serum AFP level. Similar to serum AFP level, which has been identified as a significant prognostic marker in HCC, fibrinogen may also achieve similar accuracy for predicting clinical outcomes. In fact, $\mathrm{Fu}$ et $\mathrm{a}^{33}$ found that subgroups with negative AFP levels or 
A Disease-free/recurrence-free survival

Study
$\mathrm{HR}(95 \% \mathrm{Cl}) \quad$ Weight

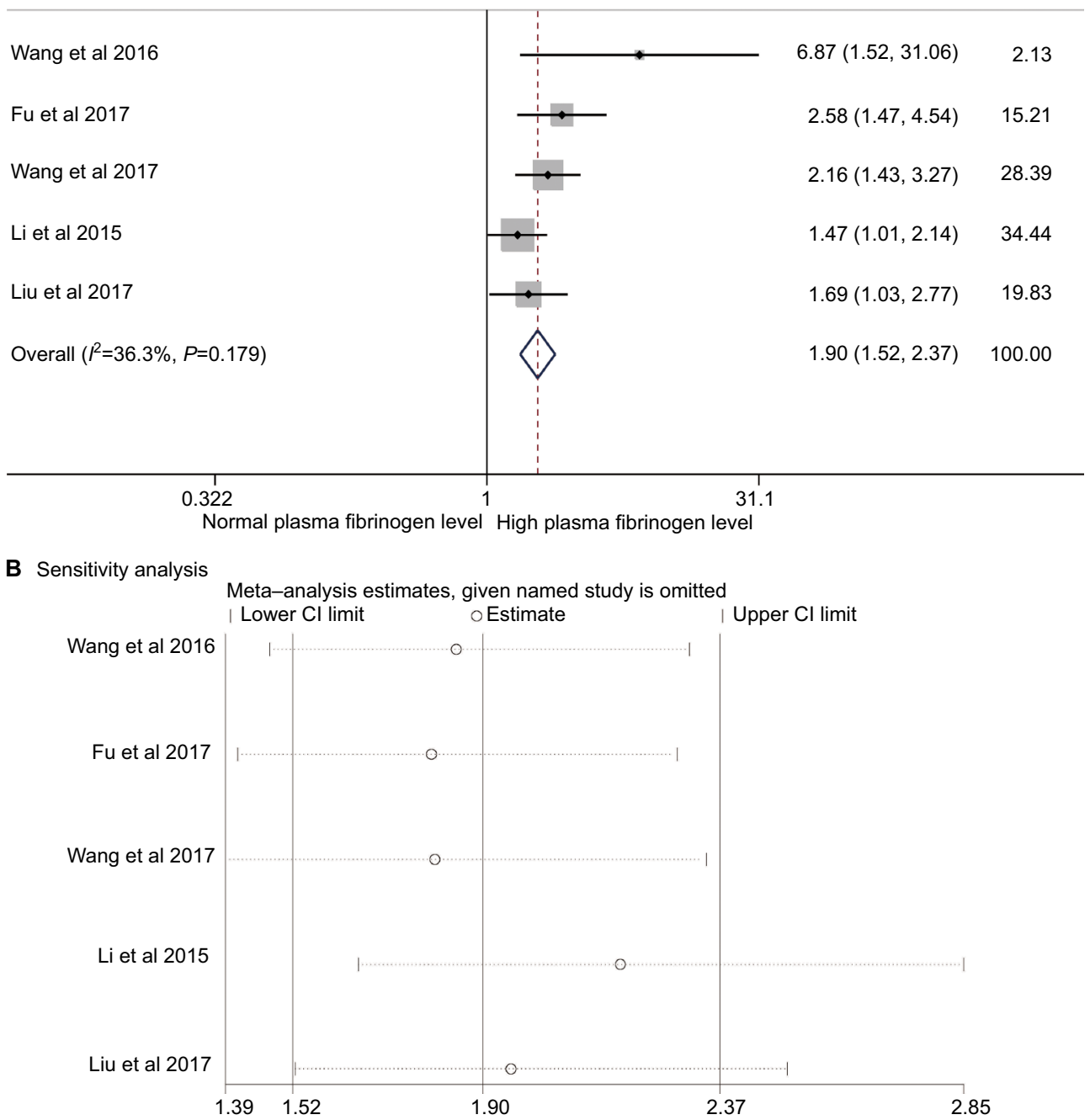

Figure 5 Association of elevated plasma fibrinogen level in patients with hepatocellular carcinoma and disease-free/recurrence-free survival. Notes: (A) Forest plot of HR for disease-free/recurrence-free survival. (B) Sensitivity analysis of disease-free/recurrence-free survival.

less aggressive clinicopathology but with elevated plasma fibrinogen levels still demonstrated poorer survival outcomes, suggesting that plasma fibrinogen could be a better prognostic biomarker compared to AFP, although further studies are warranted.

Most importantly, the examination of pretreatment plasma fibrinogen levels may help clinicians identify and provide more aggressive treatments to the appropriate group of patients to improve clinical outcomes. By comparing the normal- and high-fibrinogen groups, Zhang and Long ${ }^{19}$ found that patients with $\mathrm{HCC}$ and elevated plasma fibrinogen levels had poorer responses to TACE, a recommended adjuvant therapy for intermediate and advanced HCC. The poor response to TACE treatment in high-fibrinogen groups requires further examination due to its small sample size, and it is not one of our primary outcomes due to the limited number of studies available.

Aside from the limited studies available, there are several other limitations in our analyses. The association between the elevated plasma fibrinogen levels and poor survival outcomes could potentially be overestimated because all included studies are published studies. Positive results are always favored for publication when compared to negative results; thus exaggerating the effect of the group tested. There are variations in the plasma fibrinogen measurement methods, the cutoff values, tumor stages, and treatments received among all included studies; however, analysis suggested little heterogeneity among studies. Moreover, all patients included in the 


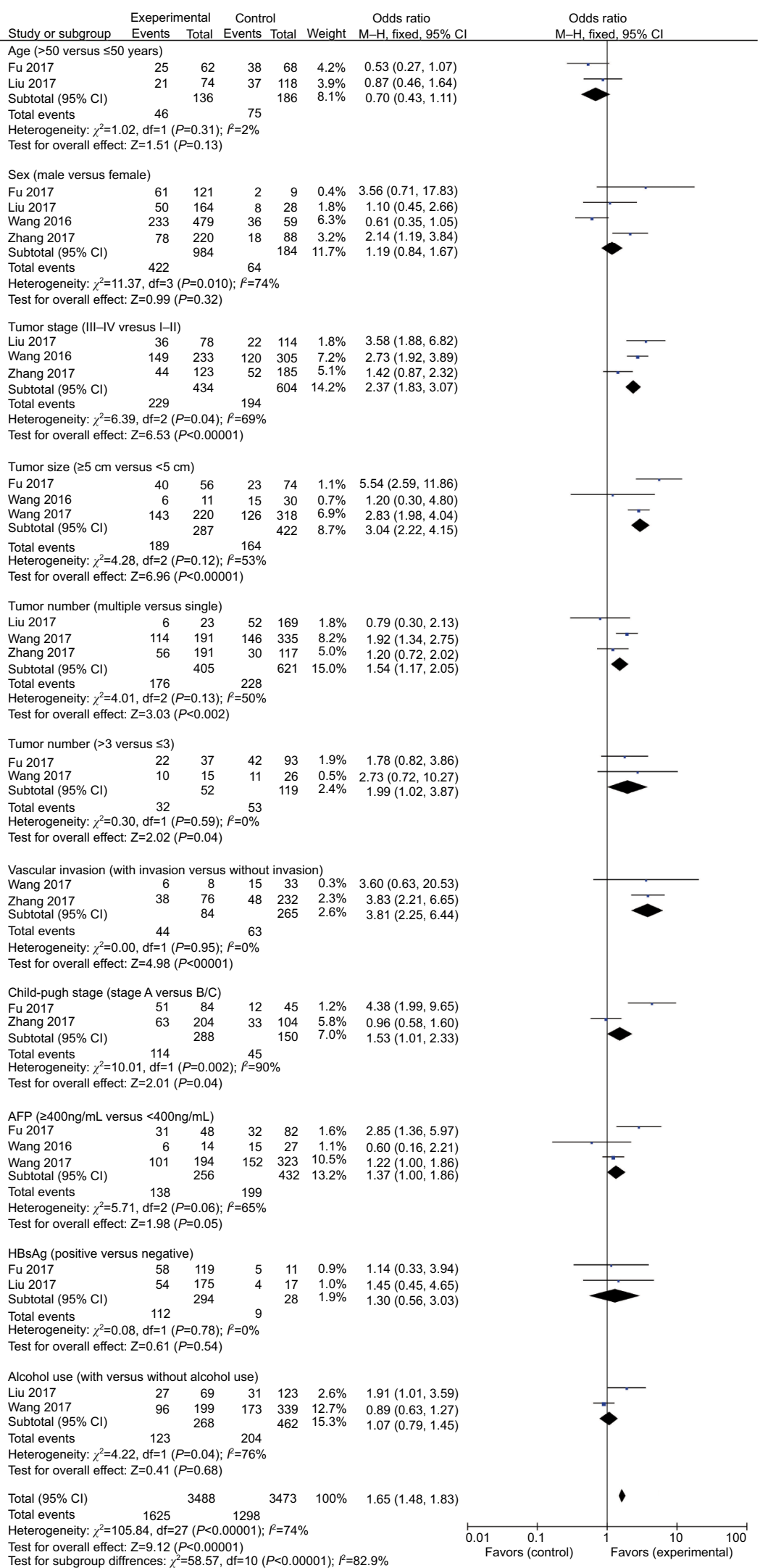

Figure 6 Association between elevated plasma fibrinogen levels and clinicopathological parameters including age ( $>50$ vs $\leq 50$ years), sex (male vs female), tumor stage (III-IV vs I-II), tumor size ( $\geq 5$ vs $<5 \mathrm{~cm}$ ), tumor number (multiple vs single; $>3$ vs $\leq 3$ ), vascular invasion (with vs without invasion), Child-Pugh stage (A vs B/C), serum AFP level ( $\geq 400 \mathrm{vs}<400 \mathrm{ng} / \mathrm{mL}$ ), $\mathrm{HBsAg}$ (positive vs negative), and alcohol use (with vs without alcohol use).

Abbreviations: AFP, alpha-fetoprotein; $\mathrm{HBsAg}$, hepatitis $B$ surface antigen. 
Table 4 Overall analysis of the association between elevated plasma fibrinogen levels and clinical features of patients with hepatocellular carcinoma

\begin{tabular}{|c|c|c|c|c|c|}
\hline \multirow[t]{2}{*}{ Variables } & \multirow[t]{2}{*}{ No. of study } & \multirow[t]{2}{*}{ OR (95\% CI) } & \multirow[t]{2}{*}{$P$-value } & \multicolumn{2}{|c|}{ Heterogeneity } \\
\hline & & & & $I^{2}(\%)$ & P-value \\
\hline Age ( $>50$ vs $\leq 50$ years $)$ & 2 & $0.70(0.43, \mathrm{I} .1 \mathrm{I})$ & 0.13 & 2 & 0.31 \\
\hline Sex (male vs female) & 4 & $\mathrm{I} .19(0.84, \mathrm{I} .67)$ & 0.32 & 74 & 0.010 \\
\hline Tumor stage (III-IV vs I-II) & 3 & $2.37(1.83,3.07)$ & $<0.00001$ & 69 & 0.04 \\
\hline Tumor size $(\geq 5$ vs $<5 \mathrm{~cm})$ & 3 & $3.04(2.22,4.15)$ & $<0.00001$ & 53 & 0.12 \\
\hline Tumor number (multiple vs single) & 3 & $\mathrm{I} .54(\mathrm{I} .17,2.05)$ & 0.002 & 50 & 0.13 \\
\hline Tumor number (>3 vs $\leq 3$ ) & 2 & $1.99(1.02,3.87)$ & 0.04 & 0 & 0.59 \\
\hline Vascular invasion (with invasion vs without invasion) & 2 & $3.81(2.25,6.44)$ & $<0.00001$ & 0 & 0.95 \\
\hline Child-Pugh (stage A vs B/C) & 2 & $\mathrm{I} .53(\mathrm{I} .0 \mathrm{I}, 2.33)$ & 0.04 & 90 & 0.002 \\
\hline AFP $(\geq 400$ vs $<400 \mathrm{ng} / \mathrm{mL})$ & 3 & $\mathrm{I} .37(\mathrm{I} .00, \mathrm{I} .86)$ & 0.05 & 65 & 0.06 \\
\hline $\mathrm{HBsAg}$ (positive vs negative) & 2 & $\mathrm{I} .30(0.56,3.03)$ & 0.54 & 0 & 0.78 \\
\hline Alcohol use (with vs without alcohol use) & 2 & $\mathrm{I} .07(0.79, \mathrm{I} .45)$ & 0.68 & 76 & 0.04 \\
\hline
\end{tabular}

Abbreviations: AFP, alpha-fetoprotein; $\mathrm{HBsAg}$, hepatitis $B$ surface antigen.

prognostic evaluation were Asians, which may not reflect as general phenomena in other ethnic populations. All of these factors could have influenced the outcome of our evaluation.

\section{Conclusion}

From our analyses of eight high-quality studies, we conclude that patients with HCC and elevated plasma fibrinogen levels have poor survival outcomes. High plasma fibrinogen levels are associated with heavier tumor burden and advanced tumor progression. Therefore, plasma fibrinogen level may serve as a prognostic biomarker in HCC.

\section{Acknowledgments}

This work was supported by the Guangdong Provincial Science and Technology Projects (No. 2017A020211031 and No. 2016ZC0145), Guangzhou Medical University Science and Technology Project (No. 201624), China National Natural Science Fund Project (81673206), Key Scientific and Technological Program of Guangzhou City (201508020262), and Guangdong Provincial Medical Science Funding Project (No. A2015243).

\section{Author contributions}

$\mathrm{GH}, \mathrm{HJ}, \mathrm{ZJ}$, and $\mathrm{XZ}$ conceived and designed the study. $\mathrm{GH}, \mathrm{HJ}$, and YL searched the database and checked them according to the eligible criteria and exclusion criteria. YW and WC analyzed the data. BS and YL designed the methodology. ZJ and XZ supervised the planning and execution of the research activity. GH and HJ wrote the paper. All authors contributed toward data analysis, drafting and critically revising the paper and agree to be accountable for all aspects of the work. All authors read and approved the final manuscript.

\section{Disclosure}

The authors report no conflicts of interest in this work.

\section{References}

1. Mazzanti R, Arena U, Tassi R. Hepatocellular carcinoma: where are we? World J Exp Med. 2016;6(1):21-36.

2. Cicalese L. Hepatocellular Carcinoma. Available from: https://emedicine. medscape.com/article/197319-overview. Accessed January 24, 2018.

3. Bosch FX, Ribes J, Díaz M, Cléries R. Primary liver cancer: worldwide incidence and trends. Gastroenterology. 2004;127(5 Suppl 1):S5-S16.

4. Zhang DY, Friedman SL. Fibrosis-dependent mechanisms of hepatocarcinogenesis. Hepatology. 2012;56(2):769-775.

5. Donato F, Tagger A, Gelatti U, et al. Alcohol and hepatocellular carcinoma: the effect of lifetime intake and hepatitis virus infections in men and women. Am J Epidemiol. 2002;155(4):323-331.

6. Huitzil-Melendez FD, Capanu M, O'Reilly EM, et al. Advanced hepatocellular carcinoma: which staging systems best predict prognosis? J Clin Oncol. 2010;28(17):2889-2895.

7. Mazzaferro V, Regalia E, Doci R, et al. Liver transplantation for the treatment of small hepatocellular carcinomas in patients with cirrhosis. N Engl J Med. 1996;334(11):693-700.

8. de Sio I, Castellano L, Calandra M, del Vecchio-Blanco C. Subcutaneous needle-tract seeding after fine needle aspiration biopsy of pancreatic liver metastasis. Eur J Ultrasound. 2002;15(1-2):65-68.

9. Chauhan R, Lahiri N, Tissue- LN. Tissue- and serum-associated biomarkers of hepatocellular carcinoma. Biomark Cancer. 2016;8(Suppl 1):37-55.

10. Wang CS, Lin CL, Lee HC, et al. Usefulness of serum des-gammacarboxy prothrombin in detection of hepatocellular carcinoma. World J Gastroenterol. 2005;11(39):6115-6119.

11. Mosesson MW. Fibrinogen and fibrin structure and functions. JThromb Haemost. 2005;3(8):1894-1904.

12. Tennent GA, Brennan SO, Stangou AJ, O’Grady J, Hawkins PN, Pepys MB. Human plasma fibrinogen is synthesized in the liver. Blood. 2007;109(5):1971-1974.

13. Wen J, Yang Y, Ye F, et al. The preoperative plasma fibrinogen level is an independent prognostic factor for overall survival of breast cancer patients who underwent surgical treatment. Breast. 2015;24(6):745-750. 
14. Shu YJ, Weng H, Bao RF, et al. Clinical and prognostic significance of preoperative plasma hyperfibrinogenemia in gallbladder cancer patients following surgical resection: a retrospective and in vitro study. $B M C$ Cancer. 2014;14:566.

15. Lee SE, Lee JH, Ryu KW, et al. Preoperative plasma fibrinogen level is a useful predictor of adjacent organ involvement in patients with advanced gastric cancer. $J$ Gastric Cancer. 2012;12(2):81-87.

16. Yamashita H, Kitayama J, Taguri M, Nagawa H. Effect of preoperative hyperfibrinogenemia on recurrence of colorectal cancer without a systemic inflammatory response. World J Surg. 2009;33(6):1298-1305.

17. Jiang HG, Li J, Shi SB, et al. Value of fibrinogen and D-dimer in predicting recurrence and metastasis after radical surgery for non-small cell lung cancer. Med Oncol. 2014;31(7):22.

18. Kinoshita A, Onoda H, Imai N, et al. Elevated plasma fibrinogen levels are associated with a poor prognosis in patients with hepatocellular carcinoma. Oncology. 2013;85(5):269-277.

19. Zhang X, Long Q. Elevated serum plasma fibrinogen is associated with advanced tumor stage and poor survival in hepatocellular carcinoma patients. Medicine. 2017;96(17):e6694.

20. Casaril M, Capra F, Marchiori L, et al. Serum copper and ceruloplasmin in early and in advanced hepatocellular carcinoma: diagnostic and prognostic relevance. Tumori. 1989;75(5):498-502.

21. Doğan UB, Cindoruk M, Dumlu S, Unlü R, Unal S. Diagnostic value of serum copper, zinc and plasma fibrinogen in cirrhotic patients with and without hepatocellular carcinoma. Ital J Gastroenterol Hepatol. 1997;29(5):476-477.

22. Zhu WL, Fan BL, Liu DL, Zhu WX. Abnormal expression of fibrinogen gamma (FGG) and plasma level of fibrinogen in patients with hepatocellular carcinoma. Anticancer Res. 2009;29(7):2531-2534.

23. Basili S, Andreozzi P, Vieri M, et al. Lipoprotein (a) serum levels in patients with hepatocarcinoma. Clin Chim Acta. 1997;262(1-2):53-60.

24. Liu Z, Guo H, Gao F, et al. Fibrinogen and D-dimer levels elevate in advanced hepatocellular carcinoma: High pretreatment fibrinogen levels predict poor outcomes. Hepatol Res. 2017;47(11):1108-1117.

25. Hessien M, Ayad M, Ibrahim WM, Ularab BI. Monitoring coagulation proteins during progression of liver disease. Indian J Clin Biochem. 2015;30(2):210-216.

26. Higgins JP, Thompson SG, Deeks JJ, Altman DG. Measuring inconsistency in meta-analyses. BMJ. 2003;327(7414):557-560.

27. Mantel N, Haenszel W. Statistical aspects of the analysis of data from retrospective studies of disease. J Natl Cancer Inst. 1959;22(4):719-748.

28. Dersimonian R, Laird N. Meta-analysis in clinical trials. Control Clin Trials. 1986;7(3):177-188.

29. Alkim H, Ayaz S, Sasmaz N, Oguz P, Sahin B. Hemostatic abnormalities in cirrhosis and tumor-related portal vein thrombosis. Clin Appl Thromb Hemost. 2012;18(4):409-415.

30. Al Ghumlas AK, Abdel Gader AG, Al Faleh FZ. Haemostatic abnormalities in liver disease: could some haemostatic tests be useful as liver function tests? Blood Coagul Fibrinolysis. 2005;16(5):329-335.

31. van der Walt JA, Gomperts ED, Kew MC. Hemostatic factors in primary hepatocellular cancer. Cancer. 1977;40(4):1593-1603.

32. Wang GY, Jiang N, Yi HM, et al. Pretransplant level is a novel prognostic predictor for hepatocellular carcinoma recurrence and patient survival following liver transplantation. Ann Transplant. 2016;21:125-130.

33. Fu SJ, Ji F, Han M, et al. Prognostic value of combined preoperative fibrinogen and neutrophil-lymphocyte ratio in patients with hepatocellular carcinoma after liver transplantation. Oncotarget. 2017;8(3):4301-4312.

34. Wang XP, Mao MJ, He ZL, et al. A retrospective discussion of the prognostic value of combining prothrombin time(PT) and fibrinogen(Fbg) in patients with hepatocellular carcinoma. JCancer. 2017;8(11):2079-2087.

35. Ruan DY, Lin ZX, Wang TT, et al. Nomogram for preoperative estimation of long-term survival of patients who underwent curative resection with hepatocellular carcinoma beyond Barcelona clinic liver cancer stage A1. Oncotarget. 2016;7(38):61378-61389.
36. Li Y, Ruan DY, Yi HM, Wang GY, Yang Y, Jiang N. A three-factor preoperative scoring model predicts risk of recurrence after liver resection or transplantation in hepatocellular carcinoma patients with preserved liver function. Hepatobiliary Pancreat Dis Int. 2015;14(5): 477-484.

37. Liu FT, Gao H, Wu CW, Zhu ZM. The association of plasma fibrinogen with clinicopathological features and prognosis in esophageal cancer patients. Oncotarget. 2017;8(54):93029-93038.

38. Lv GY, Yu Y, An L, Sun XD, Sun DW. Preoperative plasma fibrinogen is associated with poor prognosis in esophageal carcinoma: a metaanalysis. Clin Transl Oncol. 2018;20(7):853-861.

39. Matsuda S, Takeuchi H, Kawakubo H, et al. Prognostic impact of change in the fibrinogen and albumin score during preoperative treatment in esophageal cancer patients. World J Surg. 2017;41(11):2788-2795.

40. Fan S, Guan Y, Zhao G, An G. Association between plasma fibrinogen and survival in patients with small-cell lung carcinoma. Thorac Cancer. 2018;9(1):146-151.

41. Chen P, Wang C, Cheng B, et al. Plasma fibrinogen and serum albumin levels (FA score) act as a promising prognostic indicator in non-small cell lung cancer. Onco Targets Ther. 2017;10:3107-3118.

42. Cui J, Yu M, Zhang N, et al. Prognostic scores based on the preoperative plasma fibrinogen and serum albumin level as a prognostic factor in patients with upper urinary tract urothelial carcinoma. Oncotarget. 2017;8(40):68964-68973.

43. Zhang B, Song Y, Jin J, et al. Preoperative plasma fibrinogen level represents an independent prognostic factor in a Chinese cohort of patients with upper tract urothelial carcinoma. PLoS One. 2016;11(3):e0150193.

44. Luo Y, Kim HS, Kim M, Lee M, Song YS. Elevated plasma fibrinogen levels and prognosis of epithelial ovarian cancer: a cohort study and meta-analysis. J Gynecol Oncol. 2017;28(3):e36.

45. Hong T, Shen D, Chen X, Wu X, Hua D. Preoperative plasma fibrinogen, but not D-dimer might represent a prognostic factor in non-metastatic colorectal cancer: a prospective cohort study. Cancer Biomark. 2017;19(1):103-111.

46. Tian Y, Hong M, Jing S, et al. Clinical and prognostic effect of plasma fibrinogen in renal cell carcinoma: a meta-analysis. Biomed Res Int. 2017;2017:9591506-8.

47. Peng D, He ZS, Li XS, et al. A novel predictor of survival with renal cell carcinoma after nephrectomy. $J$ Endourol. 2017;31(4): 397-404.

48. Yu X, Hu F, Yao Q, Li C, Zhang H, Xue Y. Serum fibrinogen levels are positively correlated with advanced tumor stage and poor survival in patients with gastric cancer undergoing gastrectomy: a large cohort retrospective study. BMC Cancer. 2016;16:480.

49. Yu W, Wang Y, Shen B. An elevated preoperative plasma fibrinogen level is associated with poor overall survival in Chinese gastric cancer patients. Cancer Epidemiol. 2016;42:39-45.

50. Dvorak HF, Harvey VS, Estrella P, Brown LF, Mcdonagh J, Dvorak AM. Fibrin containing gels induce angiogenesis. Implications for tumor stroma generation and wound healing. Lab Invest. 1987;57(6): 673-686.

51. Sahni A, Simpson-Haidaris PJ, Sahni SK, Vaday GG, Francis CW. Fibrinogen synthesized by cancer cells augments the proliferative effect of fibroblast growth factor-2 (FGF-2). J Thromb Haemost. 2008;6(1):176-183.

52. Sahni A, Francis CW. Vascular endothelial growth factor binds to fibrinogen and fibrin and stimulates endothelial cell proliferation. Blood. 2000;96(12):3772-3778.

53. Witsch E, Sela M, Yarden Y. Roles for growth factors in cancer progression. Physiology. 2010;25(2):85-101.

54. Zheng S, Shen J, Jiao Y, et al. Platelets and fibrinogen facilitate each other in protecting tumor cells from natural killer cytotoxicity. Cancer Sci. 2009;100(5):859-865.

55. Moher D, Liberati A, Tetzlaff J, Altman DG, The PRISMA Group. Preferred Reporting Items for Systematic Reviews and Meta-Analyses: The PRISMA Statement. PLoS Med. 2009;6(7):e1000097. 
Cancer Management and Research

\section{Publish your work in this journal}

Cancer Management and Research is an international, peer-reviewed open access journal focusing on cancer research and the optimal use of preventative and integrated treatment interventions to achieve improved outcomes, enhanced survival and quality of life for the cancer patient.

The manuscript management system is completely online and includes

Submit your manuscript here: https://www.dovepress.com/cancer-management-and-research-journal

a very quick and fair peer-review system, which is all easy to use. Visit $\mathrm{http}: / / \mathrm{www}$.dovepress.com/testimonials.php to read real quotes from published authors. 